\title{
Automated Real-time Analysis of Atomic-resolution STEM Images
}

\author{
Jacob Madsen ${ }^{1 *}$, Andreas Post $1^{1}$ and Toma Susi ${ }^{1}$ \\ 1. Faculty of Physics, University of Vienna, Vienna, Austria. \\ * Corresponding author: jacob.madsen@univie.ac.at
}

Understanding the microstructural characteristics of materials, such as lattice defects, increasingly relies on the analysis of large numbers of images from electron and scanning probe microscopy [1]. It is now becoming routine to record series of atomic-resolution images, resulting in the generation of massive datasets. The new challenge is then analysing this data. The workflow for such analyses typically comprises the identification of atomic positions and subsequent derivation of physical quantities, such as defect concentration and strain. Conventionally, this analysis is done manually, which is slow and laborious, and the results are prone to human errors and bias.

We demonstrate an automatic method for extracting information from atomically resolved images. We take advantage of GPU acceleration and fast graph-based algorithms to enable real-time structural analysis. The method is capable of extracting high-level information such as defect type, lattice orientation and strain, as well as characteristics of the electron probe. Our method is based on two algorithms, building on recent advances in deep-learning and on computational geometry and graph theory.

The deep learning recognition model is similar to recently published results [2, 3]. A neural network is trained to identify the smallest distinguishable repeated substructures within the image, i.e. atoms or atomic columns of a particular species. We take advantage of the recent finding that deep neural networks trained using simulated data can generalise to experimental data [2]. Furthermore, by using randomisation in the generation of the synthetic images, the neural network is capable of making predictions with minimal prior assumptions of the types of defects present. Fig. 1 shows the results of the neural network applied to a noisy image of graphene with a silicon substitutional defect. A simple routine converts the predictions of the neural network to a set of 2D points representing the centres of the detected substructures, each point associated with a substructure class. We further explore the precision of the detected atom locations and their sensitivity to the imaging parameters including noise.

The geometric relationship between these points encodes further information, for example, whether the substitutional silicon atom in graphene has three or four carbon neighbours. To facilitate fast geometric analysis, we create a geometric graph from the detected points. It is crucial that the graph is stable to small perturbations of the atomic positions. We identified a type of geometric graph, called a stable Delaunay graph [4], fulfilling this criterion while being fast to construct for large numbers of points. Using simple rules localised segments of the graph can be extracted representing an atom and its surrounding neighbourhood. Each segment of the graph is compared to a library of known graph templates, representing, for example, different defect types. The similarity between a segment and a template is calculated using the symmetry invariant root-mean-squared-deviation [5]. After identifying the best matching template, further geometric analysis can be performed, such as calculating the strain.

The algorithm is invariant to scale and rotations, and effectively parameterless for a given set of structures, allowing it to operate without user input. Fig. 2 illustrates the algorithm applied to a set of points 
representing a grain boundary in graphene. In this example, the algorithm identifies the individual grains by their orientation and distinguishes between different types of defects along the grain boundary.

We have implemented the method in the open source microscope control software Nion Swift [6], allowing the user to overlay the results on top of the images as they are recorded, thus providing easily understandable feedback, from which the human operator can adjust the characterisations in response. Furthermore, in an effort to enable large-scale atom manipulation [7], we are working towards an increasingly self-driving microscope, where the output of the present method is used to guide the microscope to make the necessary actions, e.g. move the scan frame or the probe position, similar to the way a self-driving car would adjust the steering wheel upon encountering an obstacle on the road [8].

\section{References:}

[1] S Pennycook and P Nellist, Scanning Transmission Electron Microscopy (Springer, New York).

[2] J Madsen et al., Advanced Theory and Simulations 1 (2018), p. 1800037.

[3] M Ziatdinov et al., ACS Nano 11 (2017), p. 12742.

[4] P Agarwal et al., Discrete Computational Geometry 54 (2015), p. 905.

[5] I Kufareva and R Abagyan, Methods in Molecular Biology 857 (2012), p. 231.

[6] C Meyer et al., Proceedings of Microscopy and Microanalysis 20 (2014), p. 1108.

[7] M Tripathi et al., Nano Letters 18 (2018), p. 5319.

[8] The work was supported by the European Research Council (ERC) Grant No. 756277-ATMEN.
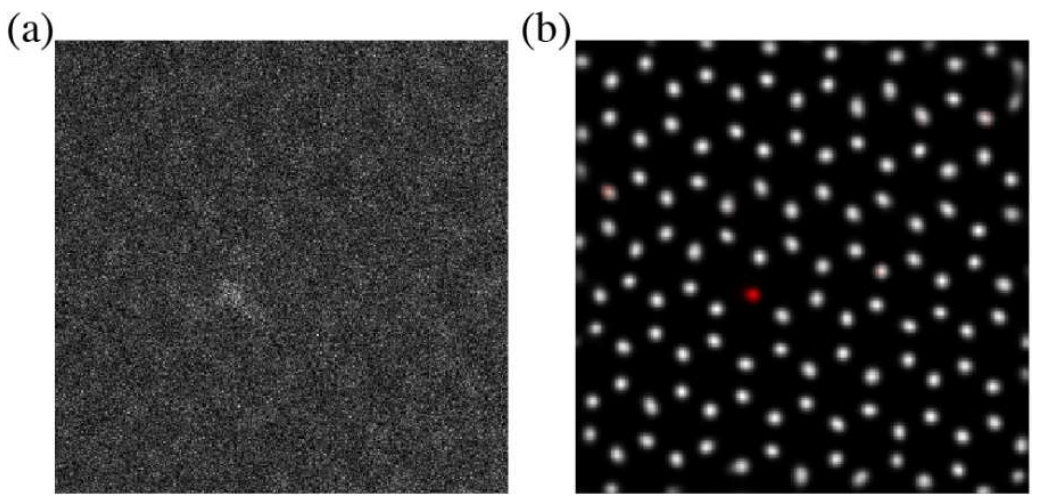

Figure 1. (a) Noisy STEM/MAADF image of graphene with a substitutional silicon defect. (b) Corresponding output of our deep learning recognition model. The white and red areas indicate positions of high likelihood for finding a carbon and a silicon atom, respectively.

(a)

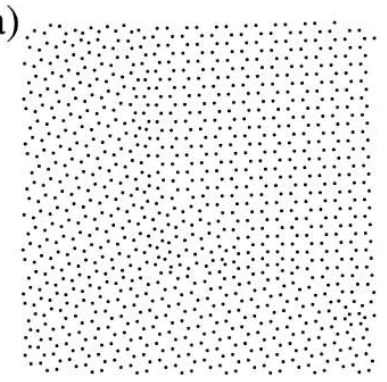

(b)

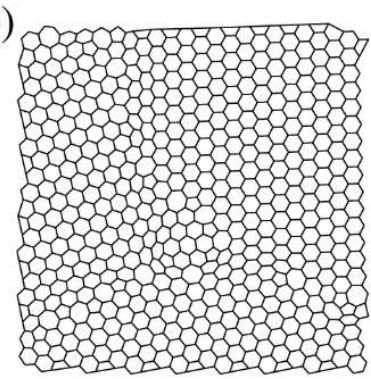

(c)

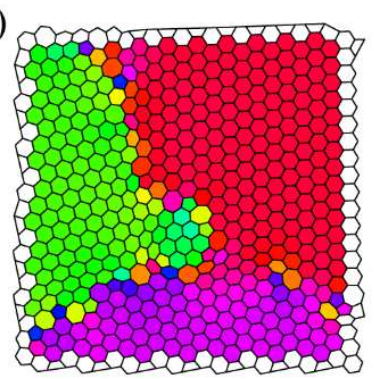

(d)

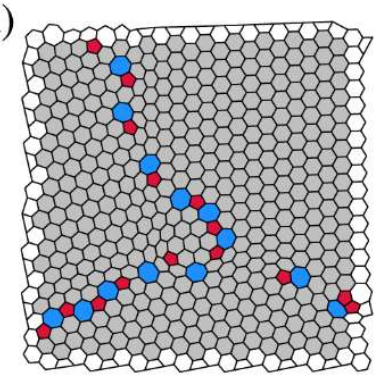

Figure 2. (a) A set of points representing the atomic positions at a grain boundary in graphene. (b) The stable Delaunay graph of the points. (c) The carbon rings of the graphene sheet are color-coded according to the orientation, assisting easy identification of different grains. (d) The carbon rings of the graphene sheet are colorcoded according to their number of members. Further graph-based analysis can be used to automatically identify for example Stone-Wales defects. 